\title{
Research on Decoding QR Code based on Image Processing Technology
}

\author{
Jianmin Guo, Lijie Feng \\ Information engineering department, Engineering College of CAPF, Xi'an, 710086, China
}

Keywords: two-dimensional code, QR code, image processing, Decoding.

\begin{abstract}
In allusion to the problem of poor encoding effect of the recognition of QR Code restricted by image acquisition devices and affected by the interference of some external factors, the decoding method after pre-processing the image of QR code by image processing technology is studied in this paper. Experiments show that, this method can effectively remove the interference from background and noise, achieving the purpose of accurate, rapid recognition and better decoding effect.
\end{abstract}

\section{Introduction}

The bar code technology has 60 years' history. The information of one-dimensional bar code is limited by capacity, so only to identify items, but not to describe them. At the same time, onedimensional bar code must rely on computer networks and back-end database, so in the environment that network resources are restricted or not easy to build a database, the onedimensional bar code is difficult to come in handy. So the two-dimensional code will come into being [1] because of huge information capacity, wide range of representation, good expression on anti-fake, secrecy and error correction ability.

There are more than 20 species two-dimensional codes that widely used, such as PDF417, QR code, Code49. QR code which is designed by Japanese companies in 1994 is a two-dimensional matrix code [2]. Because the QR code has ultra-high speed read, error correction ability strong, unique optimization processing functions for Chinese character information, accord with Chinese national habits [3-4]. Therefore, QR codes in our country has a broad application prospects, the study of the QR code recognition technology has far-reaching significance.

The application of two-dimensional code is the comprehensive application of set of barcode image recognition, coding and decoding rules and bar code printing technology in one. This article is to solve the problem that two-dimensional code recognition in the decoding process will be damaged, hardware performance differences of the collection of the equipment itself, the acquisition of the two-dimensional code image will appear distorted, tilt, uneven illumination and other issues, through image processing technology to reduce the influence of above factors on the barcode decoding, improve the decoding efficiency of QR code.

\section{Image structure of $Q R$ code}

Each QR code symbol consists of a square array of square blocks. It is composed of coding regions and function patterns including finder pattern, separator, timing pattern and correct graphic. Function patterns are not used for data coding and the sides of the symbol are blank areas. Figure 1 shows the structure chart of symbols of QR code in version 7 [5]. 


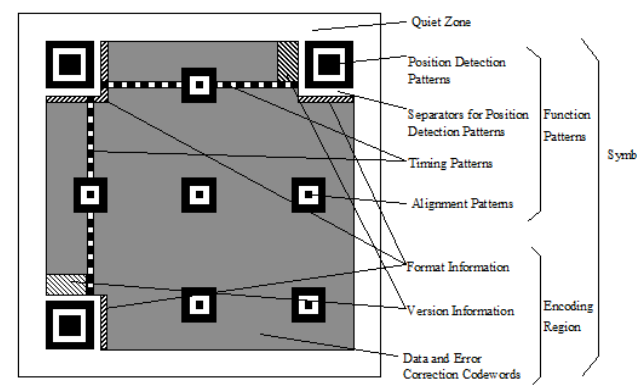

Fig.1 Structure chart of symbols of QR code in version 7

\section{Image processing course of $Q R$ code}

\section{Image gray processing}

Normally collected images are colour image, colour image contains large amount of information, the amount of storage space, and the colour image processing is relatively complex, the computation is big also, in order to simplify the process, colour information must be projected to the gravy space, so first of all to grayscale[6] of the QR code image. Gary calculation formula is as follows: $I=0.3 R+0.59 G+0.11 B$ ( $I$ is subjective colour effects, $R 、 G 、 B$ are red, green and blue components, respectively).

\section{Image median filtering}

The two-dimensional code in the process of acquisition is often disturbed by various noises, the image has edge burr and isolated point noise. In order to improve the decoding efficiency of twodimensional code, should carry on the filter demonising processing. Through the verification, the median filtering is a better demonising method, can eliminate the noise while better protect image edge. The window size and shape of two dimensional median filter have great effect. If the window is bigger, it will loss of a collection of small feature and edge information. Since the basic module of QR code image is square, so using a square window will have better results. Determined by experiment, using median filter template for 3x3 square window works best. Figure 2 for unfiltered barcode image, figure 3 is the effect after using $3 \times 3$ window filter.

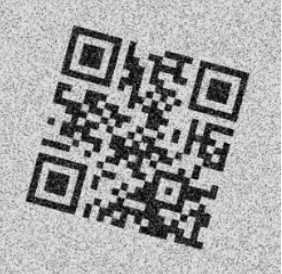

Fig. 2 Original image

\section{Image binaryzation}

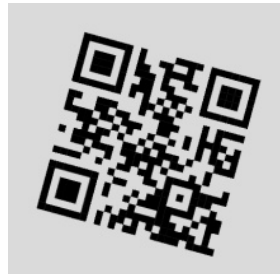

Fig. 3 Filtering image of 3x3 rectangular window

Image binaryzation refers to the threshold theory to use the method of dot operator in image processing course to change the 256 colours gravy image into white-ground and black-ground binary image, which provides favourable conditions for the image analysis, in this paper, the optimal threshold value method is selected to conduct the image processing to grayscale images.

The image contains the target gravy value and the background gravy value, s(z) expresses the sum of unimodal density functions of target and background. Formula 1 is mixed probability density with additive Gaussian noise

$$
s(z)=S_{1} s_{1}(z)+S_{2} s_{2}(z)=\frac{s_{1}}{\sqrt{2 \pi} \sigma_{1}} \exp \left[-\frac{\left(z-\mu_{1}\right)^{2}}{2 \sigma_{1}^{2}}\right]+\frac{s_{2}}{\sqrt{2 \pi} \sigma_{2}} \exp \left[-\frac{\left(z-\mu_{2}\right)^{2}}{2 \sigma_{2}^{2}}\right]
$$

Formula 1 has 5 unknown parameters, including: prior probability based on gravy value of background region $S_{1}$, prior probability based on gravy value of target region $S_{2}, S_{1}+S_{2}=1$; mean square error about mean value in background region $\sigma_{1}$, mean square error about mean value in 
target region $\sigma_{2}$; average regional value in background region $\mu_{1}$, average regional value in target region $\mu_{2}$, and Texpresses the threshold value, as shown in Fig 4.

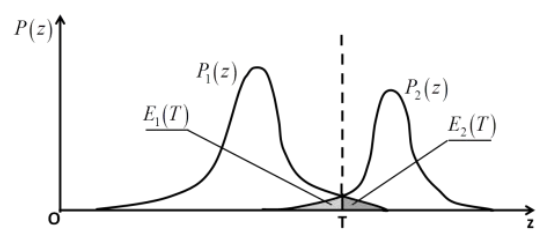

Fig. 4 Example to select the optimal threshold

Formula 2 stands for background pixel probability:

Formula 3 stands for the target pixel probability:

$$
E_{1}(T)=\int_{-\infty}^{T} s_{2} d z
$$

$$
E_{2}(T)=\int_{-\infty}^{T} s_{1} d z
$$

Formula 4 stands for the total error probability:

$$
E(T)=S_{2} \times E_{1}(T)+S_{1} \times E_{2}(T)
$$

When $\frac{\partial E(T)}{\partial T}=0$ the minimum error threshold value of formula 4 can be calculated, and the following formula is obtained

$$
S_{1} \times S_{1}(T)=S_{2} \times S_{2}(T)
$$

By substituting formula 1 into it, we get the quadratic formula:

$$
\left\{\begin{array}{l}
A=\sigma_{1}^{2}-\sigma_{2}^{2} \\
B=2\left(\mu_{1} \sigma_{2}^{2}-\mu_{2} \sigma_{1}^{2}\right) \\
C=\sigma_{1}^{2} \mu_{2}^{2}-\sigma_{2}^{2} \mu_{1}^{2}+2 \sigma_{1}^{2} \sigma_{2}^{2} \ln \left(\frac{S_{2}}{S_{1}}\right)
\end{array}\right.
$$

In general, if $\mu_{1}=\mu_{2}$, only an optimal threshold value can be calculated:

$$
T_{\text {optimal }}=\frac{\mu_{1}+\mu_{2}}{2}+\frac{\sigma^{2}}{\mu_{1}-\mu_{2}} \ln \left(\frac{S_{2}}{S_{1}}\right)
$$

When $\mathrm{S}_{1}=\mathrm{S}_{2}$, the optimal threshold value can be calculated:

$$
T_{\text {optimal }}=\frac{\mu_{1}+\mu_{2}}{2}
$$

In formula 9, the 5 unknown parameters in s(z) can be obtained:

$$
e_{m s}=\frac{1}{n} \sum_{i=1}^{n}\left[s\left(z_{i}\right)-h\left(z_{i}\right)\right]^{2}
$$

In formula 9, $\mathrm{n}$ is a gravy level, $\mathrm{h}(\mathrm{z})$ is a function, and $\mathrm{s}(\mathrm{z})$ is a density function of mixed probability. The image effect is better after being processed by the optimal threshold value, as shown in Fig 5.

\section{Edge extraction}

Edge detection is often carried out by the use of spatial differential operator to the convolution of its templates with images. The gay level edge is the result of discontinuous gay value, such a discontinuity can normally be detected by using the differentiation conveniently. In general, firstorder derivative and second-order derivative are used to detect the edge. Gradient corresponds to the first-order derivative, and gradient operator is the first-order derivative's operator, when the grey value filtering of edge is sharper and the image noise is smaller, the work efficiency of gradient operator is better.

For a continuous image function $\mathrm{f}(\mathrm{x}, \mathrm{y})$, its gradient in the position $(\mathrm{x}, \mathrm{y})$ is expressed as vector $\nabla f(x, y)=\left[G_{x}, G_{y}\right]^{T}=\left[\frac{\partial f}{\partial x}, \frac{\partial f}{\partial y}\right]^{T}$, in which $G_{x}$ and $G_{y}$ respectively stand for the gradients along $\mathrm{X}$ direction and $\mathrm{Y}$ direction. The magnitude and direction angle of this vector respectively are $\operatorname{mag}(\nabla f)=\left(G_{x}^{2}+G_{y}^{2}\right)^{\frac{1}{2}}, \phi(x, y)=\arctan \left(G_{x} / G_{y}\right)$, in which the amplitude is calculated by taking 2 as the template, 
other templates can be used to calculate the magnitude for the sake of the convenient calculation, while for the above partial derivatives the position of each pixel should be calculated. Each template should be used for $G_{x}$ and $G_{y}$, each separately, so two templates should be combined to form a gradient operator. According to the different template size and element values, people have put forward many different operators, such as Prewitt operator, sober operator and Kirsch operator. After verification, sober operator has better effect, and the results after edge extraction is as shown in Fig 6.

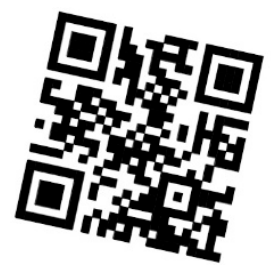

Fig. 5 Image after binaryzation

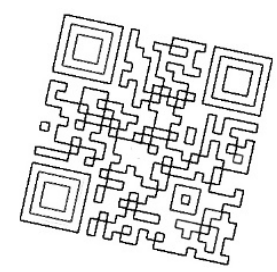

Fig. 6 Edge detection diagram

\section{Image rotation}

QR code has the characteristic of 360 degree recognition, so rectification should not be required by a tilted image in theory. But if tilting rectification isn't required by an image, the specific position and tilt angle of symbols of QR code should be solved by using position detection pattern's character array of symbols of QR code to match the templates, which will take a lot of computing time. In this paper, the method of rotation first and then recognition is adopted to rapidly identify the images without tilting, which saves the time from template matching and tilt angle solving, saves the extra overhead of the recognition of tilting images, and reduces the difficulty for subsequent decoding.

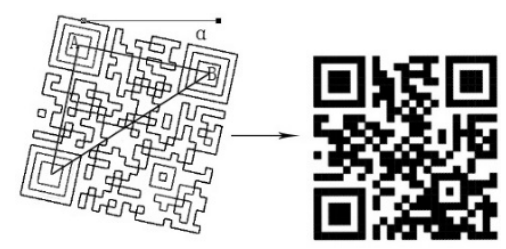

Fig. 7 Rotated image

Because the position detection pattern has a special proportion, meanwhile under the action of intermediate photomask, the pattern with such a proportion may not occur in other positions of QR codes, therefore, the bar code can be accurately positioned by finding the three position detection patterns. In addition, whether the bar code need to be rotated and the rotation angle can be determined through centre coordinates of the three position detection patterns. If the rotation is required by the image, please rotate the image to the standard position as shown in Fig 7 . Before rotating the image, firstly please determine the rotation angle of image $\alpha$ [7], by setting the coordinates of two points A, Bin the image as $A\left(X_{A}, Y_{A}\right)$ and $B\left(X_{B}, Y_{B}\right)$, we get $\tan \alpha=\frac{Y_{A}-Y_{B}}{X_{B}-X_{A}}$, then we can also obtain the value of $\alpha$.

\section{Decoding QR code}

After image pre-processing, the symbol pattern is converted to a light and dark matrix array, decoding as follows [8]:

(1) Determine the finding patterns according to the edge detection algorithm described in the above.

(2) Determine the position distribution of the three position detection patterns according to the coordinates of centre points of position detection patterns. Then determine the distance between position detection patterns as shown in Fig 8. D is the width between the two position detection 
patterns above the symbol patterns, $\mathrm{W}_{\mathrm{UL}}$ and $\mathrm{W}_{\mathrm{UR}}$ are respectively the width of modules of the two position detection patterns.

(3) Determine the width of the basic module of symbol patterns according to formula 10:

$$
X=\left(W_{U L}+W_{U R}\right) / 14
$$

(4) Determine the version number of symbol patterns according to formula 11 :

$$
V=[(D / X)-10] / 4
$$

(5) If symbol patterns' version number $\geq 7$, the decoding steps of symbol patterns' version information are as follows:

a. The width of the basic module of symbol patterns according to formula 12:

$$
C P=W_{U R} / 7
$$

b. As shown in Fig 9, the straight lines $A B, A C$ are the link lines of the centre of position detection patterns, the version information 1 and 2 contain sampling grid of the centre of each modules. According to the coordinates of centre points of straight lines $\mathrm{AB}, \mathrm{AC}$, module width $\mathrm{CP}$ and finding patters, sampling grids of version information 1 can be determined.

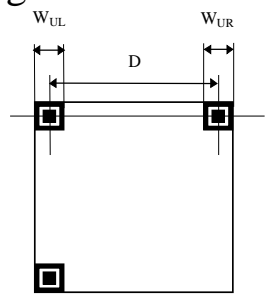

Fig. 8 Width between detection patterns

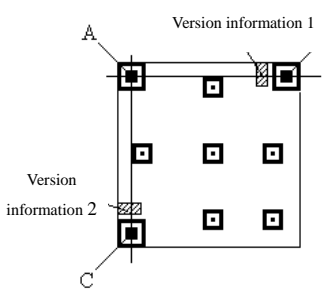

Fig. 9 Version information

c. Determine the version information of symbol patters according to encoding specification of version information and RS correct error code principle. If there are too many errors during the process of detection and error correction, please decode the data information in version information 2.

(6) Determine the sampling grids according to the coordinates of centre points of alignment patters, the sampling grid of alignment patters is as shown in Fig 10.

a. Calculate the width of the basic module of symbol patterns according to formula 13, in which $\mathrm{W}_{\mathrm{UL}}$ is the width of position detection patterns,

$$
C P_{U L}=W_{U L} / 7
$$

b. As shown in Fig 10, P1and $\mathrm{P}_{2}$ are two alignment patters in symbol patterns, whose centre modules are located in the link lines between the centres of position detection patterns, preliminarily determine the coordinates of centre points of $\mathrm{P}_{1}$ and $\mathrm{P}_{2}$ according to the width of the basic module of symbol patterns.

c. Determine the actual coordinates of centre points of alignment patterns according to the range of light modules in $\mathrm{P}_{1}$ and $\mathrm{P}_{2}$, after the coordinates of centre points of and are determined, as shown in Fig 11.

d. Preliminarily determine the coordinate of centre point of alignment pattern $\mathrm{P}_{3}$.

e. Repeat the step c to determine the actual coordinate of centre point of alignment pattern $\mathrm{P}_{3}$.

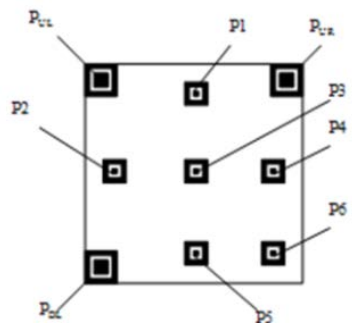

Fig. 10 Sampling grids of alignment patterns 


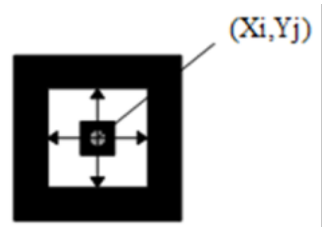

Fig. 11 Center coordinates

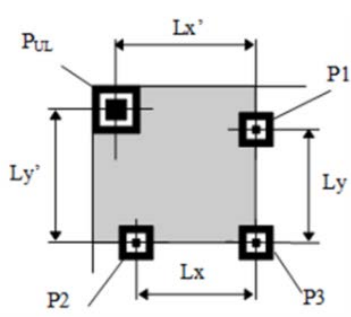

Fig. 12 Upper-left area

of alignment patterns of symbol patterns

f. Determines the distance between alignment patterns, as shown in Fig12, $\mathrm{L}_{\mathrm{x}}$ and $\mathrm{L}_{\mathrm{y}}$ are the distance between centre modules of alignment patterns $\mathrm{P}_{1}, \mathrm{P}_{2}$ and $\mathrm{P}_{3}$.

g. Determine sampling grids of alignment patterns and determine sampling grids at the upper-left area of symbol patterns according to the distance between the centres of alignment patterns.

h. Repeat the above steps to determine sampling grids at the lower-right area and uncovered area of symbol patterns.

(7) Firstly, detact and determine the error correction level and photomask pattern reference according to the format information beside the separator at upper-right area of position detection patterns. If the total errors are bigger than its error correction capacity during the detection process, please decode the format information area in other positions.

(8) Remove the photomask and carry out the Exclusive OR to symbol patterns of photomask pattern reference and coding region to obtain the required data code word sequences.

(9) Permute and combine the data code word sequences in blocks according to error correction level and symbolic versions.

(10) Correct the non-read and substitution errors in data code word according to RS errorcorrection decoding algorithm.

(11) Convert the data code word permutation and combination in blocks into original data code word sequence.

(12) Decode each section of data code word sequence according to each section of data code word's mode indicator and character count indicator.

\section{Conclusion}

In this paper, QR code image pre-processing technology and QR code decoding method are studied, wherein the image pre-processing includes image greying, binaryzation, filtering and noise reduction, edge detection and image rotation, which has realized the recognition of QR code in practical application. Experiments show that, QR codes decoding method based on image processing technology can effectively identify the contaminated, anamorphic and tilting bar codes, which thus has broad application prospects.

\section{References}

[1]Pavlidis T, Swartz J. Fundamentals of barcode information theory [J]. IEEE Transactions on Computers, Vol. 23(1990) No.4, p. 74.

[2]The National Standard of the People's Republic of China GB / T18284--2000. Quick Response Code [s].

[3]Ottaviani E, Pavan A, et al. A common image processing framework for 2D barcode reading. Image Processing and its Applications, Conference Publication, IEE, 1999 (465): p.652-655

[4]Liu Yue, Liu Mingye, Liu Mingjun. Design of Automatic Recognition Algorithm of Quick Response Code. Computer Systems \& Applications, 2006 (6)

[5]Chinese Standard Press. Compilation of National Standards of Barcode [M]. Beijing: China Standard Press, 2004 
[6]Ouyang Wei. Research and Implementation of QR Code Automatic Identification System [D]. Shanghai: Fudan University, 2006

[7]Wan Qing. Coding and Decoding and System Implementation of QR Code [D]. Shanghai: Encoding and decoding system and two-dimensional code realization [D]. Shanghai: Shanghai Jiao Tong University, 2007

[8]Zhao Qunli, Zhou Qiuping, Shi Junhua. Discussion on Image Recognition Technology of QR Code. Journal of Hefei Normal University, 2013, 31 (3): p. 24-26. 